\title{
Sustainable institution of post-event reconstruction in developing countries: defining the non-engineered construction paradigm
}

\author{
S. A. Akbar ${ }^{1}$, M. Siddiq ${ }^{2}$, M. U. M. Awan ${ }^{1}$, S. Shah ${ }^{1} \&$ M. Iqbal ${ }^{1}$ \\ ${ }^{I}$ Department of Architecture, University of Engineering and Technology, \\ Lahore, Pakistan \\ ${ }^{2}$ Xi Etui Architectural Research, Lahore, Pakistan
}

\begin{abstract}
In the developing world, despite official promotion and support, engineered structures continue to fail to take root in post-event reconstruction efforts. Both the $2005 \mathrm{Kashmir}$ and 2001 Gujarat earthquakes are excellent case studies of owner preference in this regard where the overwhelming majority of reconstructed building stock turned out to be non-engineered.

Based upon first hand surveys of reconstruction after the Kashmir earthquake and other published studies, this paper attempts to analyse aspects of nonengineered construction that tend to make it more desirable to owners as compared with the engineered options. It finds that these aspects can be divided into two categories. The first one, not entirely specific to non-engineered structures, relates to aspects of physical design such as construction materials and techniques, as well as aesthetic and cultural aspects.

The second and critically important aspect is the construction paradigm of the non-engineered structure which takes place within the larger construction milieu of the region. The paper analyses and contrasts the construction paradigm of both types of structures and identifies it as the key difference between the two. It further concludes that improving seismic performance of the non-engineered structure is strongly dependent upon respecting its construction paradigm, and that any change in the culture of construction must be brought about through it.

Keywords: construction paradigm, non-engineered structures, seismic performance, post-event reconstruction, owner driven reconstruction.
\end{abstract}




\section{Introduction: the case for non-engineered structures}

The silver lining to destruction caused by a large earthquake is the opportunity to reduce the risk of death and destruction for a future disaster through improving seismic performance of the replacement building stock, and instituting improved construction practices in the process. Results of reconstruction efforts, however, differ widely around the globe with much of the developing world failing to institute improved construction techniques in their reconstructed building stock, as shown by Abidi et al. [1] in an overview of post-event reconstruction in four Asian countries. Many other studies including UN-HABITAT [10] support this conclusion.

It is maintained that a major reason for this disappointing performance by the developing countries lies with the solution strategies that assume presence of an engineered construction paradigm. Since the engineered construction paradigm does not exist in developing countries [8], the reconstruction drive fails to produce the expected results and the improved construction techniques introduced as part of the reconstruction drive fizzle out as the subsidies dry up.

\subsection{Can't see the wood for the trees}

The design and construction of individual structures does not take place in vacuum. There is always a supporting milieu in the form of an extensive infrastructure that consists of architects, engineers, construction craftsmen and technologists, building materials and components, business and market mechanisms related to construction, as well as education and training infrastructure catering to the needs of building industry. It also includes local and higher level governmental and non-governmental structures and regulatory agencies that set up standards and minimum acceptable levels of safety, and ensure that building practices conform to the desired minimum.

It is this larger milieu that shapes individual structures, quite like the way the jungle generates and shapes individual trees. When an earthquake strikes an area, it destroys individual structures but this larger milieu, the construction paradigm of the region stays the same. New reconstructions would invariably be shaped by it. Efforts to the contrary including subsidies for reconstruction can only go a limited way. As the intervention dies out, the 'old ways' re-emerge, for they are inevitable products of the larger infrastructure.

A review of literature reveals that in this particular regard, the inability to see the wood for the trees is rather universal. A large body of works exists that is aimed at improvements in the building construction practices of the developing world such as IAEE guidelines for non-engineered construction [5]. Such improvements, however, are typically suggested without any reference to the regional construction milieu, or the local construction paradigm. Indeed many times these are suggested with an implicit assumption that a fully regulated engineered construction paradigm is in place or that if missing, one shall be put in place. 
This approach is effectively equivalent to proposing the institution of new species of trees while assuming that the jungle shall be altered to suit the requirements of the new species. Even if we agree that the new species of trees is desirable and the jungle needs to be altered, it cannot be achieved overnight. In stark contradiction, however, post-event reconstruction does need to take place 'overnight.'

In practical terms, however, a radical change in the local non-engineered construction paradigm (jungle) is rarely needed. Slight changes in the existing construction paradigm may well be sufficient to institute construction systems with adequate seismic performance, as long as the proposed construction systems are designed for the existing non-engineered construction paradigm. This is supported by an increasing number of studies such as $[2,13]$. In terms of the jungle analogy, it is being suggested that the new species should not be alien to the local environment; rather they should be modifications of well acclimatized local species. It is, thus, maintained that in the case of developing countries, improvements in local building practices can be sustainably instituted if such improvements are designed to work within the non-engineered construction paradigm of the region in question.

\subsection{Adequacy for post-event reconstruction}

Engineered structures are, by definition, individually custom designed by a licensed professional engineer. A non-engineered structure, on the other hand, belongs to the class of construction where the owner, following the constructional norms of his region, builds a structure employing builders familiar with that constructional norm. The only 'engineering' incorporated within a nonengineered structure is through the 'training' and 'experience' of builders employed for the construction, supplemented by the knowledge and experience of the owner and his friends and relatives.

This is, however, not to say that the structure is likely to be structurally unsound. The construction 'norms' of a region typically have roots extending back through several generations, often comprising of centuries of common experience of structural performance of 'norms.' Thus there is always a process of gradual improvement of the constructional practices in areas where it is found to be deficient. A common experience of a whole community, thus, tends to perfect its constructional norms over a long period of time. From a scientific point of view, this 'empirical' approach towards 'engineering' a structure is essentially sound $[1,2,14]$.

Enhancing seismic performance of the reconstructed building stock can be further ensured through a regulated regime for reconstruction aimed at institution of improved construction techniques [11]. This should help achieve minimum standards for structural performance, public health, services, and contextual relationships.

In this sense, these buildings are engineered albeit with the distinction that these are engineered as a class rather than individually. The effectiveness of such engineering, especially for residential structures is beyond question. Indeed 
typical wood-frame single residence structures in Japan as well as in California USA continue to be non-engineered and offer excellent seismic performance.

\subsection{Why non-engineered construction?}

The post-event reconstruction of both 2001 Gujarat $[11,16]$ and 2005 Kashmir earthquake [2] consisted almost exclusively of non-engineered structures. The experience of these owner driven reconstruction efforts clearly indicates that people tend to favour them. It is important to understand why these are favoured, for therein lays the key to intervene and institute improvements in the popular construction practices, and this constitutes the primary subject matter of this paper. In order to avoid complexity, the paper involves itself exclusively with residential structures. The discussion and analysis, however, will be equally applicable to non-residential structures as well.

The following Section provides a methodical description of characteristics of non-engineered construction, and juxtaposes it with the engineered structures, effectively laying down a set of criteria for design of a constructional system suitable for adoption in a zone of significant seismic risk. This analysis is based upon both a number of post-disaster surveys of reconstruction in the Kashmir, Hazara and Sawat-Dir regions of Northern Pakistan some of which are referenced at [6-9], as well as on a large number of published studies such as [4, $12,15,16]$.

\section{Characteristics of non-engineered constructional systems}

Non-engineered constructional systems differ from the engineered structures in multiple aspects only some of which are technical in nature. Other and more important aspects are based in the structure and state of development of the society and its culture. Still others are related to the process of construction rather than to the constructed object. For these reasons it may well be maintained that the human factor is more dominant in the design of non-engineered structures than technical factor $[12,16]$.

\subsection{The primary paradigm}

The primary paradigm within which an engineered structure takes shape has four components: Client, Consultant, Contractor, and Project. A client means an owner who is willing to pay the consultants for design and to a professional contractor to execute the project, and has access to sufficient funds to complete the project within a reasonably contiguous time-frame. In the non-engineered paradigm, the owner has no professional consultants, the professional contractor is replaced by local builders, and the construction project is typically an openended construction activity mainly due to paucity of funds. Owner typically lives in the under-construction structure, and additions are made as and when possible, sometimes after long intervals.

This change of paradigm has strong implications upon design of the constructional system. For instance nobody in the owner's team may have the 
ability to read technical drawings, let alone implement them. Thus the nonengineered constructional system needs to be expressed in terms communicable to the 'owner.' Similarly materials and techniques that require highly skilled manpower or strong on-site quality control may not be suitable for nonengineered construction. Quite obviously, the regulation regime will also have to be different for the two classes of construction. Further implications of this shift in the primary paradigm and the way it tend to turn the non-engineered structure into an entity quite different and distinct from the engineered structures are expanded on in the following sections.

\subsection{Cultural acceptability of construction system is the key}

In an engineered structure, the client is well advised about available options for the project and his cultural preferences are embodied within the design of the structure. The client thus finalizes his choices and the design formally completed before the construction begins. In the case of non-engineered construction individual building is never formally designed. As such, the choice before the owner is not about design of his house, but about adoption of a constructional system for his house. Thus the deciding factor would be cultural acceptability of the constructional system as perceived by the owner rather than design of his individual house.

\subsection{Suitable only for single residences}

Low technical expertise on the part of the 'owner-builder' team means that nonengineered constructional system is not entirely suitable for structures that require skilled manpower, special construction equipment, or high technical accuracy. These requirements are typical of 'larger' projects. Thus it is advisable that only small residential projects (1-2 story single family residence) are allowed under the ambit of non-engineered construction. Similarly materials or construction techniques that require strong on-site quality control e.g. concrete frame construction should also not be allowed under the non-engineered regime, even for 'small' projects.

\subsection{The owner is anonymous}

In the engineered construction regime the owner is well identified. Consultants interact with him personally and his requirements and preferences find priority place in the designs. In the non-engineered regime, identifiability of the owner as an individual is largely irrelevant and meaningless, often not possible as well. His meaningful identity is as a member of a group or section of the society. Thus accommodating the requirements, desires and preferences of the owners within the design can only be accommodated through multiple options only. It is thus highly desirable that the range of options available to the owner is maximized.

One desirable strategy is to customize the constructional system for each region by accommodating its popular construction materials and techniques, as well as its cultural peculiarities. Thus the proposed system should be, in terms of 
available options, as near as possible to the time tested existing system because the existing set of practices embody local culture and preferences to a maximum extent. Similarly all subgroups and communities within the society must also be reflected in the proposed regime.

\subsection{Architectural design is a continuous process}

Irrespective of whether a professional architect is employed, the architectural design of engineered structures is fully determined and technical drawings are completed before any engineering design is taken up. The architectural design of a non-engineered structure however is typically defined by the owner himself; any drawing is at best a crude sketch. Oftentimes even important components such as the location of an aperture, and occasionally even the location of an adjacent room, are decided after construction has started. This very fluid design process indicates that many post construction changes may also be made. Indeed a residence may not be completely built in one go with additions made to it horizontally or vertically as changes within the family dictate or economic conditions permit. The proposed construction system should be designed to accommodate such post-construction changes and piecemeal design process.

\subsection{Engineering design is always implicit}

As noted previously, engineered structures go through customized engineering design individually. So should the non-engineered construction system, but as a class. In the case of engineered structures, the design is specified through a set of documents in the form of technical drawings and specifications. In the case of non-engineered construction, the design must also be specified but mainly expressed as building code. Additional non-binding information may be produced as supporting documents. Another method of design specification is through ensuring quality components through the construction market and specifying their use.

\subsection{Construction process is non-contiguous}

Engineered structures are characterized by professionally executed construction in a well defined contiguous project. Professional contractors are involved, and sufficient funds are available to ensure uninterrupted construction. In any case, the client typically steps in only after the construction is complete in all respects.

Construction of non-engineered structures is typically owner organized, who engages various builders for specific construction tasks. Typically multiple builders are involved, and construction is a long drawn process. Shortage of funds or other reasons may cause construction to be taken up in spurts, many times separated by long intervals. Similarly part of the structure may be occupied by the owner while the rest of construction is going on. In short, construction of non-engineered structures is many times far from the 'clean' construction process of engineered structures. Thus the proposed constructional system should be designed to gracefully accommodate alterations and additions. 


\subsection{Regulatory mechanisms must be different}

That the local regulatory body shall play its role is equal in both instances. Beyond that, the regulatory mechanisms for the two classes of structures are diametrically opposite in their principal approach.

In the case of engineered structures, the primary role in regulation and quality assurance is played by licensed professionals. The law assumes that professional architects, engineers, and construction professionals shall perform their tasks adequately. For instance, that a licensed engineer has designed the structure is considered adequate assurance of good engineering design. The primary paradigm of the engineered structure also contains inherent characteristics to assure adequate quality of construction. The professional consultants act as agents of the owner, keeping a check on the contractor and (by default) on each other. This crosscheck essentially constitutes the self-regulation of engineered construction.

The non-engineered construction process, on the other hand does not enjoy any similar crosscheck. The builder is also the primary advisor of the owner. Owner being bereft of any professional advice, quality of construction is essentially subjected to the professional competence (or lack thereof) and motives of the builders. Self-regulation, therefore, is all but absent from the nonengineered construction paradigm.

This imbalance in non-engineered construction paradigm calls for additional regulatory measures. To begin with, the role of regulatory bodies must be more vigorous with checks and appraisals carried out at several stages of design, construction and even post-construction. Second, builders must be professionally trained and certified. These and other such initiatives (as mentioned below) are a need for the construction industry (including both engineered and nonengineered structures) and as such their utility will be universal.

\subsection{Builder training is a must}

The two classes of construction also differ acutely vis-à-vis construction skills. Engineered projects are far fewer in number than non-engineered ones, and also offer significantly better profit margins. Professional contractors are thus able to train their construction workers to their own standards, and ensure reasonable workmanship. In any case, any non-native building skills can always be imported from elsewhere where needed.

In the absence of professional contractors, and facing dearth of funds, nonengineered segment cannot benefit from a similar strategy. In its case, the abundant native building skills must be available. It is thus mandatory for any successful regime of non-engineered construction that builders and building trade craftsmen are trained in a certification program in abundant numbers to serve the entire region. There is no alternate to this measure. 


\subsection{Quality assurance through building materials and components}

The case of building materials and components is quite similar to that of skills. For engineered structures, required building materials and components can be ordered or imported if not available locally. In any case higher ability to pay and professional handling of construction preclude use of sub-standard quality materials and components.

The non-engineered segment, on the other hand, has to use what is available in the nearest market, often paying a price similar to a higher quality component. This 'natural' tendency of markets (and the buyer) to gravitate towards lower quality levels has already been mentioned above. It is essential that safeguards against this trend are instituted and minimum quality standards are enforced. To this end, both certification at the manufacturer level and licensing at the wholesale and retail levels in the local market must be ensured.

\subsection{Vulnerabilities}

In the case of engineered structures, the primary vulnerability lies with its reliance upon self-regulation, dependence upon competence and ethical standards of consultants and contractors. Regulatory mechanisms for both, in the form of quality education, training, and professional institutes with regulatory roles are a must.

Non-engineered construction, on the other hand, suffers from low technical expertise at the construction site. Thus any materials or construction techniques that require high on-site quality control are likely to induce vulnerability. RCC Frame construction, for instance, proved to be a major contributor to the death toll in the October 2005 earthquake where substandard construction led to its exceptionally low performance. Such materials and techniques must be restricted to the engineered segment, and must not be permitted (unregulated) in the nonengineered construction.

\subsection{Institution of change}

Finally both classes of construction also differ in their acceptance of a change in regulatory laws. The engineered segment effectively responds to any change in underlying laws. Thus a change, say in required structural performance or measures against fire hazard would be immediately accepted. This is largely because the owner is highly identifiable, and also because of economic impetus of the project which imbues it with a sense of urgency.

The non-engineered segment, on the other hand, behaves in a different manner. Law, typically, proves to be quite ineffective due to large number of owners which provides a certain degree of anonymity to any particular individual. Corrupt practices in the law enforcement personnel also find a shield in large numbers. Finally the 'public' also has a political say and nuisance value which deters any strong action by regulatory agencies. 
However, it must be recognized that all fair systems must be based upon choice, and not coercion. Thus any institution of change in the non-engineered section must be gradual, and primarily geared towards public acceptance.

\section{Barriers to popular acceptance}

Architecture of a people is one of the most visible and enduring of their cultural expressions. Popular building materials and construction techniques are a key medium of architectural expression and an integral component of the larger culture. As such, it is deep rooted and requires sophisticated and subtle approach for any intervention.

In order to institute any changes, it is essential that the existing incarnations of the culture of construction are well identified. In particular its geographical expanse, its variations as they relate to various socio-economic or other layers within the society, and its historical origins must be clearly identified. Furthermore the design of the strategy for change must accept that change shall set in gradually. In particular, it should be sensitive to the aspects mentioned in the following which act as barriers to public acceptance of changes in construction technology. Divided into three groups, these do not form a comprehensive listing, but are just indicative of their kind.

\subsection{Technological and functional}

As indicated in the preceding sections, non-engineered segment of construction operates with low technical know-how and professional expertise. Typically builders or other building craftsmen bring not much of tools or machinery, except their own personal skills. Thus construction processes that require significant construction machinery would not find favour with the builders.

Similarly techniques requiring high degree of precision, ability to read technical drawings and knowledge of English technical terminology obviously require properly trained and professionally skilled professionals that are difficult to come-by in the non-engineered segment and would constitute a barrier to their popular usage. Similarly materials or finishes that are difficult to repair or those requiring frequent maintenance are also not likely to find favour with this segment of construction.

\subsection{Economics and marketing}

The non-engineered segment essentially functions off the local market. What is not available in the nearest building materials store is not likely to be used. Thus desirable building materials or components must be available widely. The same is also true about availability of skills. The owner is the chief organizer of construction, and quite like finances, short on available effort. Thus the owner will likely seek to employ the builder next door for next tranche of construction. Similarly, a skill not available locally will be a skill not employed, or shunned. For the same reasons, overall cost, especially perceived value for money will always be a prime factor in construction related decision-making. 
As it can be sensed from the preceding, there is a strong tendency to gravitate towards lower cost hence lower quality, both in terms of materials as well as workmanship. The legal 'stick' or even a financial 'carrot' in the form of a reconstruction subsidy would only go a limited way towards correcting this 'natural' bias.

An increase in the general levels of education and economic prosperity are essential to counter this trend. Until then, though, training and education of the builders who act as principal construction advisors of the owner is vital. Professional advice from 'Building Advice Centres' is also certain to be valued by owners, and helpful in checking this trend.

\subsection{Human and cultural}

This is certainly the most important category in this section, and vital for the success of any program aimed at upgradation of non-engineered segment. Man being what he is, develops emotional ties even with inanimate objects that include building materials and finishes. Thus familiarity with a building material, especially one with deep historical roots produces strong bias in its favour.

In fact it is not difficult to continue to employ building materials traditional to an area. Sometimes it can be achieved with a change of role (from structural to an infill panel use, or as a finishing veneer), or a change in the construction technique might provide the requisite improvement (timber post-and-lintel construction changed to timber frame).

Familiarity, in fact, is not just surface deep. It also embodies experience of using that material, knowing techniques of its maintenance, and an understanding of its vulnerabilities and strengths. And of course its popularity also ensures that local craftsmen understand it, and appropriate building skills for its use abound. Finally it also takes the form of confidence in its functional performance so necessary for its popular employment, and owners shall not need to be pushed and cajoled towards its employment.

Unfamiliar materials may also be perceived as lacking value for money, especially if they are radically different from the prior experience of the society. One example is that of modern low density lightweight insulation materials which strongly contrast the traditional thick stone walls in the Kashmir region used for their insulation value.

However, there is one factor that has an overriding effect upon practically all other factors mentioned above, viz. the perceived status of a construction system. People would be willing to pay a higher than usual cost for a construction system that is perceived to carry status. For instance in the Kashmir region, burnt bricks, hugely more expensive than the local stone (or concrete block), was a preferred construction material because it carried a higher status due of its association with Islamabad, the capital city!

This can be used as a powerful tool for introduction of new technologies. One technique to 'engineer' status for any new constructional system is by its employment in important government building such as courts, schools, hospitals, and police stations etc. 


\section{Concluding remarks}

In the preceding sections, a case has been built up in favour of employing the non-engineered segment of constructional practice as the favoured construction segment for reconstruction of single family residential structures in developing countries. It has been argued that non-engineered construction can in fact be engineered to a desired level of structural or seismic performance, and that these are inherently suited for large scale owner-driven reconstruction efforts.

It was shown that a major reason for failure of engineered construction to take root in many developing countries is the regional construction milieu of these countries which strongly favoured the non-engineered construction. It thus follows that the key to instituting any improvements in the culture of construction of any region is to bring about such changes through its larger construction milieu; that engineered solutions, however good they might be, shall not find favour with population at large in their own right.

In support of this contention, a detailed analysis was presented which shows that the fundamental paradigm for engineered structures is radically different from that of the non-engineered structures. This analysis was then used to lay down the basis for instituting changes in the prevalent construction practices aimed at improving the seismic performance of the reconstructed building stock.

Finally it is pointed out that sustainable institution of improvements in the culture of construction of any region is possible only through gradual changes in its larger construction milieu. Once instituted, such improvements shall continue to be practiced beyond the reconstruction phase, and shall continue to improve the seismic performance of entire building stock of the region.

\section{References}

[1] Abidi, S.R.T.Z., Akbar, S.A. \& Bioret, F., Post-event reconstruction in Asia since 1999: an overview focusing on the social and cultural characteristics of Asian countries. Proc. of the International Conference on Earthquake Engineering and Seismology (ICEES 2011), ed. Bhatti, A.Q., School of Civil \& Environmental Engineering NUST: Islamabad, pp. 418-424

[2] Khan, H.M., Shah, A., Jan, I. \& Khan, R., Evaluation and improvement of seismic performance of locally constructed non-engineered buildings in the earthquake prone areas of Pakistan. Proc. of the International Conference on Earthquake Engineering and Seismology (ICEES 2011), ed. Bhatti, A.Q., SCEE NUST: Islamabad, pp. 248-255

[3] Mumtaz, H., Mughal, S.H., Stephenson, M., \& Bothara, J.K., The challenges of reconstruction after the October 2005 Kashmir earthquake, Proc. of the 2008 NZSEE Conference, http://db.nzsee.org.nz/ 2008/Paper34.pdf

[4] Karunasena, G. \& Rameezdeen, R., Post-disaster housing reconstruction: Comparative study of donor vs. owner-driven approaches, International Journal of Disaster Resilience in the Built Environment, 1(2), pp.173 - 191, 2010 
[5] International Association For Earthquake Engineering, Guidelines for Earthquake Resistant Non-Engineered Construction, National Information Center of Earthquake Engineering, IIT Kanpur, 2004

[6] Akbar, S.A. \& Mukhtar, A., The Hindu Kush Earthquake of January 31, 1991: Post Disaster Survey and Preliminary Analysis of Vulnerability, Report No. Arch/91-01, University of Engineering and Technology Lahore, 1991.

[7] Akbar, S.A. \& Chaudhary, M.N., The Kashmir-Hazara Earthquake of October 8, 2005: Analysis of the Disaster and Strategies for Reconstruction and Rehabilitation, Report No. Arch/05-01, University of Engineering and Technology Lahore, 2005.

[8] Akbar, S.A., Kashmir-Hazara earthquake of 8th October 2005: Engineering non-engineered structures for post-disaster reconstruction, Proc. of the 4th International Civil Engineering Congress (4th ICEC-2006), Institution of Engineers Pakistan, 2006.

[9] Akbar, S.A., Awan, M.U.M., Shah, S., Iqbal, M., Evaluation of Post-event Reconstruction Drive in the Affected Region of October 2005 Kashmir Earthquake, Unpublished manuscript based upon field survey findings, 2010.

[10] UN-HABITAT, Housing Reconstruction Field Assessment: Balochistan Earthquake Affected Areas, 2010.

[11] Barenstein, J.D., Housing Reconstruction in Post Earthquake Gujarat: A comparative Analysis, HPN, Overseas Development Institute, U.K. www.odihpn.org/documents/networkpaper054.pdf

[12] Jha, A.K., Barenstein, J.D., Phelps, P.M., Pittet, D. \& Sena, S., Safer Homes Stronger Communities: A Handbook for Reconstructing after Natural Disasters, The World Bank, 2010.

[13] Pooyan, Z., \& Ghayamghamian, M.R., Sustainable Disaster Recovery: Principles for Post-quake Planning, International Disaster and Risk Conference IDRC Davos 2010, https:/www.conftool.com/idrc2010 /index.php/Pooyan-Sustainable_Disaster_Recovery-613.pdf

[14] Boen, T., Pribadi, K.S., Engineering the Non Engineered Houses for Better Earthquake Resistance in Indonesia, http://drh.bosai.go.jp/files /6cc5597e09050a9b482d9f257c5f256ec28f6e50/7_PT8_P.pdf

[15] Boen, T., Earthquake Resistant Design of Non-Engineered Buildings In Indonesia, EQTAP Workshop IV, Kamakura, 2001, http://eqtap.edm.bosai.go.jp/publications/EqTAP_ws/ws_4th/pdf/cdrom/session_2/2_21_boen/paper_2_21.pdf

[16] Barenstein, J.D. \& Iyengar, S., India: From a culture of housing to a philosophy of reconstruction (Chapter 7). Building Back Better: Delivering People-Centred Housing Reconstruction at Scale, eds. M. Lyons, T. Schilderman \& C. Boano, Practical Action Publishing Ltd.: Bourton on Dunsmore, Rugby, pp. 163-190, 2010. http:/www.ucl.ac.uk/dpu/people /boano/tabs/building-back-better-book.pdf 